\title{
Síndrome de Burnout em acadêmicos de Medicina
}

\author{
Burnout Syndrome in Medicine academics \\ Síndrome de Burnout en estudiantes de Medicina
}

Recebido: 06/04/2021 | Revisado: 12/04/2021 | Aceito: 17/04/2021 | Publicado: 27/04/2021

\author{
Luzilene Pereira de Lima \\ ORCID: https://orcid.org/0000-0003-1038-2711 \\ Universidade Católica de Pernambuco, Brasil \\ E-mail: luzilene.1011@gmail.com \\ Mayana Aquino Correia de Lima \\ ORCID: https://orcid.org/0000-0002-6419-2924 \\ Universidade Católica de Pernambuco, Brasil \\ E-mail: mayana.2019230032@unicap.br \\ Dáfiny Guimel Valéria Machado Borges \\ ORCID: https://orcid.org/0000-0002-6740-6801 \\ Universidade Católica de Pernambuco, Brasil \\ E-mail: dafiny.2019230050@unicap.br \\ Jorge Luiz da Silva Segundo \\ ORCID: https://orcid.org/0000-0002-3891-9288 \\ Universidade Católica de Pernambuco, Brasil \\ E-mail: jorge.2019203044@unicap.br \\ Pedro Henrique Bezerra de Fraga \\ ORCID: https://orcid.org/0000-0002-0994-1267 \\ Universidade Católica de Pernambuco, Brasil \\ E-mail: pedroxfraga@gmail.com \\ Rodrigo Vinícius de Lima e Silva \\ ORCID: https://orcid.org/0000-0002-7741-1874 \\ Universidade Católica de Pernambuco, Brasil \\ E-mail: rodrigo.2019203198@unicap.br \\ Victor Marcus de Menezes \\ ORCID: https://orcid.org/0000-0002-2786-4189 \\ Universidade Católica de Pernambuco, Brasil \\ E-mail: victormarcus715@gmail.com \\ Manuela Barbosa Rodrigues de Souza \\ ORCID: https://orcid.org/0000-0002-7773-100X \\ Universidade Católica de Pernambuco, Brasil \\ E-mail: manu.brsouza@gmail.com
}

\section{Resumo}

A Síndrome de Burnout (SB) é um tripé de exaustão emocional, despersonalização e falta de realização profissional, sua causa e origem é vista como multifatorial. No meio acadêmico, geralmente, as vítimas dessa síndrome são os estudantes, mais especificamente os de medicina, em virtude da tensão por cumprir as demandas do curso. Dessa forma, se buscou estabelecer a problemática da SB no acadêmico de medicina através da revisão integrativa. A organização do estudo foi por meio de pesquisas em plataformas de periódicos científicos, mapeando as informações. Não existe uma causa principal definida para o distúrbio, mas a soma de múltiplos elementos facilitam para o desencadeamento da SB, sejam fatores extrínsecos e intrínsecos. Pode ter início na época da faculdade de medicina, prossegue durante toda a residência e cresce na rotina de médicos em atividade. Por fim, o acadêmico de medicina enfrenta uma grade curricular extensa com meio intenso de produtividade, o que gera um esgotamento físico e mental durante a sua formação, além da cobrança ao estudante em ser e portar-se como um bom profissional.

Palavras-chave: Saúde mental; Burnout; Estudantes de medicina.

\begin{abstract}
Burnout Syndrome (BS) is a tripod of emotional exhaustion, depersonalization and lack of professional fulfillment, its cause and origin are multifactorial. In the university, the victims of this syndrome are usually the students, more specifically those of medicine, due to the tension of fulfilling the demands of the course. Thus, we sought to establish the problem of BS in medical students through the integrative review. The study was organized through research on scientific journal platforms, mapping the information. There is no defined main cause for the disorder, but the sum of multiple elements facilitates the onset of BS, whether extrinsic or intrinsic factors. It can start at the time of medical school, continues throughout the residency, and grows in the routine of active physicians. Finally, the medical student faces an extensive curriculum with an intense means of productivity, which generates physical and mental exhaustion during their training, in addition to charging the student to be and behave like a good professional.
\end{abstract}

Keywords: Mental health; Burnout; Medical students. 


\section{Resumen}

El Síndrome de Burnout (SB) es un trípode de agotamiento emocional, despersonalización y falta de realización profesional, su causa y origen se percibe como multifactorial. En el ámbito académico, las víctimas de este síndrome suelen ser los estudiantes, más concretamente los de medicina, por la tensión de cumplir con las exigencias del curso. Así, buscamos establecer el problema de la SB en estudiantes de medicina a través de la revisión integradora. El estudio se organizó mediante la investigación en plataformas de revistas científicas, mapeando la información. No existe una causa principal definida para el trastorno, pero la suma de múltiples elementos facilita la aparición de SB, ya sean factores extrínsecos o intrínsecos. Puede comenzar en el momento de la escuela de medicina, continúa durante toda la residencia y crece en la rutina de los médicos en activo. Finalmente, el estudiante de medicina se enfrenta a un extenso plan de estudios con un intenso medio de productividad, que genera agotamiento físico y mental durante su formación, además de cobrar al estudiante por ser y comportarse como un buen profesional.

Palabras clave: Salud mental; Burnout; Estudiantes de medicina.

\section{Introdução}

A Síndrome de Burnout (SB) tem derivação do Inglês to burn out que em tradução significa queimar-se. Foi definida por Herbert Freudenberger em 1974, um psicanalista que se autodiagnosticou como portador desta síndrome. Em 1999, Christina Maslach e Michael Leiter definiram a SB como um tripé composto por exaustão emocional, despersonalização e falta de realização profissional (Moreira, Souza e Yamaguchi, 2018).

Mori, Valente e Nascimento (2012) consideram a causa da SB de origem multifatorial, esclarecendo que no meio acadêmico, mais especificamente em alunos da graduação de medicina, é grande a pressão para mostrar-se como bons profissionais para a sociedade, amigos e familiares. Justamente por isso, o meio intenso de produtividade, muitas vezes tornam esses estudantes vítimas de Burnout.

Esta síndrome começou a ser estudada na década de 70, nos Estados Unidos, difundindo-se mundialmente nos anos posteriores. O artigo que provocou maior impacto e propagação do Burnout foi o de Freudenberger em 1974, conforme citado anteriormente, levando muitos pesquisadores a estudarem seus sintomas, causas e consequências (Benevides-Pereira, 2012).

Dessa maneira, a tensão para cumprir todas as demandas do curso de medicina, a carga horária extensa, a sobrecarga de informação, a idealização da importância do médico e do estudante de medicina podem induzir os estudantes a inibir todas as suas iniciativas de gratificação e autoindulgência (Gonçalves e Benevides-Pereira, 2009). Assim sendo, o curso de medicina provoca mudanças no estilo de vida do estudante, que na maioria das vezes têm diminuída sua qualidade de vida (Fiedler, 2008).

As fontes de tensão no curso médico podem gerar crises adaptativas e serem prejudiciais à saúde dos estudantes, dependendo de diversos fatores, entre eles as atitudes - percepções, pensamentos, crenças, valores e tendências de comportamento dos mesmos frente aos agentes estressores (Silva et al., 2009).

Ao se deparar com tantas dificuldades, o estudante é afetado por insegurança, cansaço, tristeza. Ainda assim, não deve se deixar abater: além de todos os obstáculos que deve transpor, o futuro médico precisa aprender a se portar de modo a corresponder às demandas dos professores, dos colegas e da sociedade. Há exigência por um profissional totalmente comprometido, capaz de manter a calma e a sanidade em situações adversas, além de, muitas vezes, sacrificar a própria condição de vida a fim de se dedicar à de outros (Feodrippe et al., 2013).

Nessa conjuntura, o presente trabalho visa estabelecer a problemática da SB que acomete os acadêmicos de medicina e como isto reflete no seu esgotamento físico e mental, levando o fato do mesmo estar em uma situação de forte pressão, acúmulo de atividades além de conhecimentos que precisam ser adquiridos devido à grande grade curricular, aumentando, consequentemente, a possibilidade de desenvolver esse distúrbio emocional estudado, entre os graduandos de medicina, no qual afeta severamente sua qualidade de vida e rendimento na graduação superior. Em suma, a análise e o estudo do tema são de extrema relevância frente às causas acima expostas a fim de tomar medidas preventivas e tratamentos. 


\section{Metodologia}

O presente trabalho refere-se a uma revisão integrativa, diz respeito ao método que possibilita a junção do conhecimento aliado a aplicação prática do tema, fornecendo assim informações mais amplas, de forma sistemática e abrangente sobre o problema estudado (Ercole, Melo e Alcoforado, 2014). Dessa forma, vem colaborar com a medicina baseada em evidências, através do processo de pesquisa quantitativo. As etapas que compõem a revisão integrativa são seis, descritas como: 1 - Definição: problema, estratégia de busca, descritores e bases de dados, bem como, formulação da questão guia para a pesquisa; 2 - Utilização das bases de dados e pesquisa dos estudos com foco nos critérios de inclusão e exclusão; 3 - Mapeamento das informações dos estudos pré-selecionados e selecionados; 4 - Análise crítica e categorização dos estudos incluídos; 5 - Análise e discussão dos resultados; 6 - Revisão integrativa e síntese do conhecimento com propostas para futuras pesquisas.

Primeiramente, foi identificado o tema e formulada uma questão guia para a pesquisa, sendo ela: $\mathrm{O}$ fato de estar fazendo a faculdade de medicina aumenta a possibilidade de Síndrome de Burnout nos acadêmicos?

Logo após, organizou-se a busca dos estudos nas plataformas de publicação de periódicos científicos PubMed (U.S. National Library of Medicine) e SciELO (Scientific Electronic Library Online), nos meses de outubro e novembro de 2020, com base nos critérios de inclusão e exclusão.

Os critérios de inclusão utilizados foram analisar artigos: publicados do ano de 2010 a 2020; cujo idioma seja Português ou Inglês; que seu texto completo seja disponibilizado gratuitamente; além de considerar todos os desenhos de estudo, dessa forma, não ocorreu limitação.

O critério de exclusão foi centralizado em artigos que: não sejam relacionados diretamente ao tema estudado.

Utilizou-se os seguintes Descritores em Ciências da Saúde (DeCS) na busca dos artigos: Saúde mental; Burnout e Estudantes de medicina, os correspondentes na língua inglesa são: Mental health; Burnout; Medical students.

Sendo o AND escolhido como operador booleano. Prosseguindo, foi realizado a identificação dos estudos préselecionados e selecionados, através da leitura do resumo, descritores e título das publicações, posteriormente organizando os mesmos.

Dando continuidade, o instrumento utilizado foi o Critical Appraisal Skills Programme (CASP) - adaptado, para a análise metodológica dos artigos selecionados. O CASP adaptado contempla 10 questões, citadas a seguir: 1 - Objetivo claro e conciso; 2 - Seleção apropriada de artigos; 3 - Exposição e reflexão/discussão dos procedimentos teórico-metodológicos; 4 Triagem da amostra de modo condizente; 5 - Organização de informações/dados com detalhes; 6 - Conexão do pesquisador x pesquisados levados em consideração; 7 - Dados éticos resguardados; 8 - Estudo dos dados rigoroso e embasado; 9 - Exposição e discussão dos resultados com segurança; 10 - Considerar as contribuições e limitações do estudo, bem como, sugestões para futuras pesquisas.

De acordo com as 10 questões do $C A S P$ adaptado foi organizada uma pontuação de classificação para os artigos selecionados, conforme o Quadro 1 abaixo mencionado.

Quadro 1. Pontuação de classificação dos artigos selecionados.

\begin{tabular}{|c|l|}
\hline Nível A & $6-10$ pontos (qualidade metodológica boa com baixo viés). \\
\hline Nível B & $0-5$ pontos (aceitável qualidade metodológica com risco de alto viés). \\
\hline
\end{tabular}

Fonte: Autores, (2020). 
Vale salientar que é importante observar que cada questão é avaliada com o valor zero (0) ou um (1) pontos. O resultado equivale a soma das pontuações, consequentemente o maior valor atribuído é dez (10) pontos.

\section{Resultados e Discussão}

Foram encontrados 157 artigos, 153 estão no PubMEd e 4 no SciELO, destes não atenderam aos critérios de inclusão: 17 artigos - ano de publicação (2010 a 2020); 10 artigos - idioma (Português ou Inglês); 72 artigos - texto completo (gratuito) e desenhos de estudo (todos), o que resultou em 58 artigos. Assim, 49 não estavam relacionados diretamente ao tema estudado, a amostra final se totaliza em 09 artigos (Figura 1).

No Quadro 2 é mencionado as informações mais significativas da amostra final. Relacionado ao ano de publicação, 01 artigo (11,1\%) no ano de 2015, em 2017, 01 artigo (11,1\%), já 2018, 02 artigos (22,2\%), 03 (33,3\%) em 2019 e em 2020, 02 artigos (22,2\%). Referindo-se ao idioma de publicação 08 artigos $(88,8 \%)$ estão em inglês e 01 (11,1\%) em português. 06 artigos $(66,6 \%)$ foram publicados em revistas internacionais e $03(33,3 \%)$ publicados nacionalmente. Com relação ao país de estudo, a amostra final está dividida da seguinte maneira, $06(66,6 \%)$ no Brasil, $01(11,1 \%)$ na Irlanda e 02 (22,2\%) nos Estados Unidos da América (EUA). Vale salientar, que 01 dos estudos correlacionam estudantes de três países (EUA, China e um país do Oriente Médio, cujo nome permanece anônimo por solicitação da instituição de ensino). De acordo com o $C A S P$ adaptado, o resultado foi de 09 artigos (100\%) considerados de nível A e 0 (0\%) de nível B.

Figura 1. Fluxograma de esclarecimento dos critérios utilizados e amostra final.

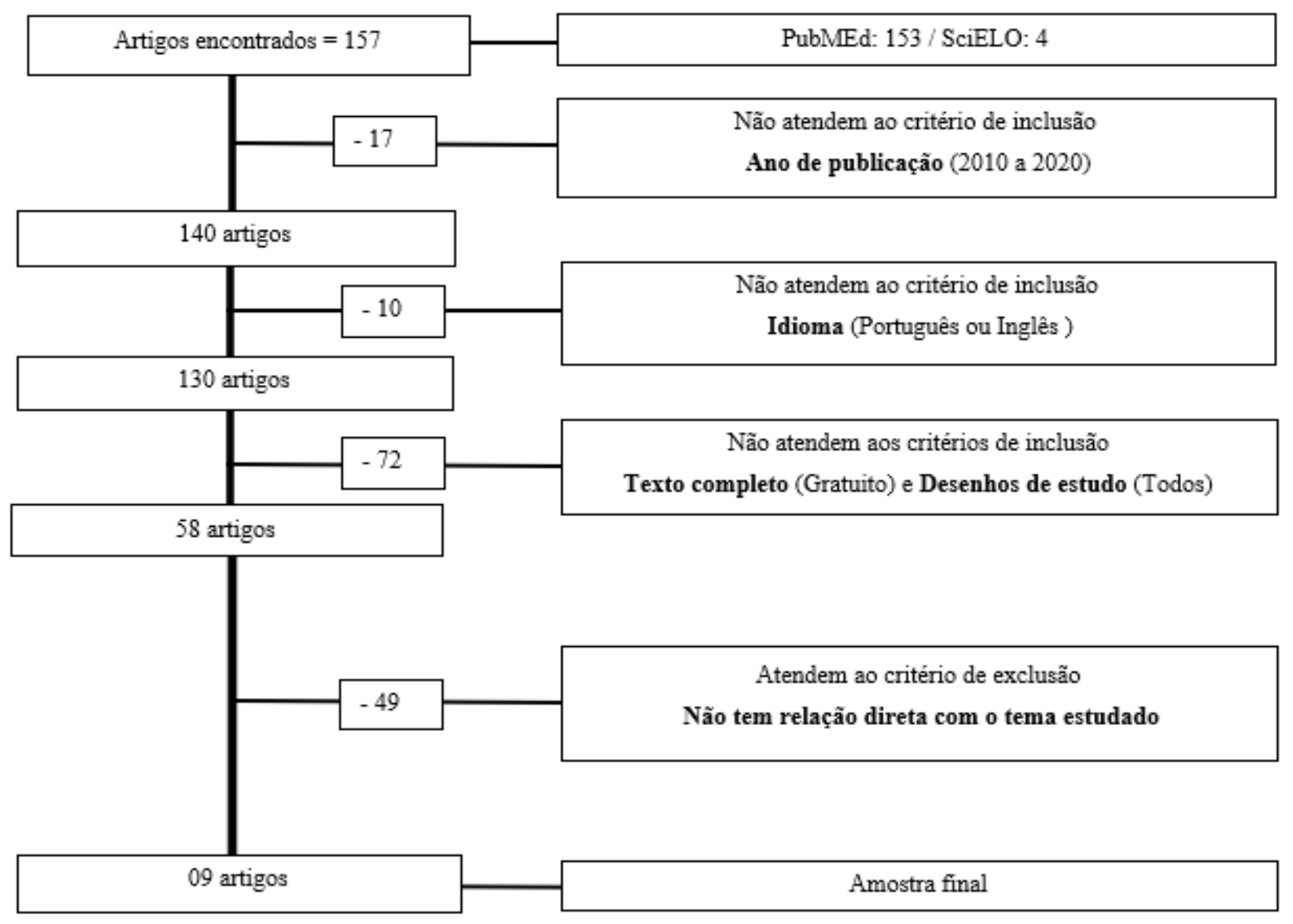

Fonte: Autores, (2020). 
Na Figura 1 se destaca os critérios que foram utilizados para selecionar a amostra final dos artigos, no início a pesquisa indicou 157 artigos e ao final, levando em consideração os critérios de inclusão e exclusão, a amostra final ficou com 09 artigos.

Quadro 2. Informações mais significativas da amostra final e nível de evidência de cada artigo, CASP adaptado. Recife - PE, 2020.

\begin{tabular}{|c|c|c|c|c|c|c|}
\hline Autor & $\begin{array}{l}\text { País de } \\
\text { estudo }\end{array}$ & $\begin{array}{c}\text { Desenho do } \\
\text { estudo }\end{array}$ & Amostra & Objetivo & Principais resultados & $\begin{array}{c}\text { Nível de } \\
\text { evidência } \\
\text { (CASP } \\
\text { adaptado) }\end{array}$ \\
\hline $\begin{array}{l}\text { Pacheco, J. } \\
\text { P. et al.; } \\
2017\end{array}$ & Brasil & $\begin{array}{l}\text { Revisão } \\
\text { sistemática e } \\
\text { meta-análise }\end{array}$ & $\begin{array}{l}18.015 \\
\text { estudantes }\end{array}$ & 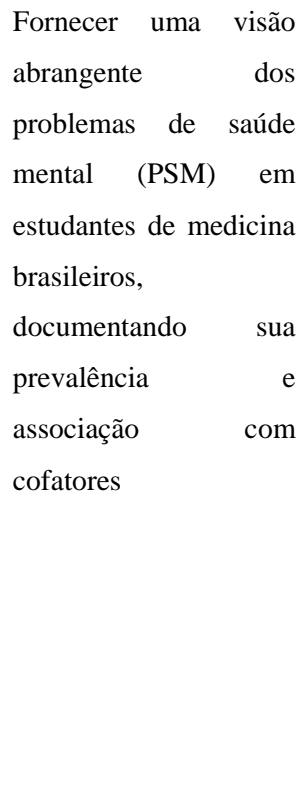 &  & A \\
\hline $\begin{array}{l}\text { Fitzpatrick } \\
\text {, O. et al.; } \\
2019\end{array}$ & Irlanda & $\begin{array}{l}\text { Transversal - } \\
\text { coorte }\end{array}$ & $\begin{array}{l}269 \text { alunos } \\
\text { pré- } \\
\text { clínicos e } \\
\text { clínicos }\end{array}$ & $\begin{array}{l}\text { Medir o Burnout e seu } \\
\text { impacto no risco de } \\
\text { depressão em uma } \\
\text { população } \\
\text { estudantes de medicina, } \\
\text { comparando os anos } \\
\text { pré-clínicos e clínicos }\end{array}$ & $\begin{array}{l}\text { Houve uma prevalência de } 39 \% \text { de casos } \\
\text { depressivos. A taxa de Burnout teve } \\
\text { mudança significativa entre os anos, } 35 \% \\
\text { com síndrome de Burnout em anos clínicos } \\
\text { e } 26 \% \text { em anos pré-clínicos. Os com poucos } \\
\text { sintomas de Burnout tiveram uma } \\
\text { prevalência de } 13 \% \text { de sintomas } \\
\text { depressivos, enquanto os de categoria } \\
\text { intermediária tiveram uma prevalência de } \\
\text { 38\% e aqueles na categoria alta tiveram uma } \\
\text { prevalência de } 66 \% \text { de sintomatologia } \\
\text { depressiva. O aumento da exaustão } \\
\text { emocional e a diminuição do rendimento } \\
\text { acadêmico aumentaram as chances de não } \\
\text { querer procurar ajuda para os problemas de } \\
\text { saúde mental }\end{array}$ & A \\
\hline $\begin{array}{l}\text { Barbosa, } \\
\text { M. L. et } \\
\text { al.; } 2018\end{array}$ & Brasil & Transversal & 399 alunos & $\begin{array}{l}\text { Identificar a } \\
\text { prevalência de Burnout } \\
\text { e fatores associados em } \\
\text { estudantes de medicina } \\
\text { brasileiros }\end{array}$ & $\begin{array}{l}12 \% \text { da amostra analisada apresentaram } \\
\text { Burnout. As mulheres com maior taxa (8\%) } \\
\text { e os homens }(4 \%) \text {. O } 3^{\circ} \text { ano teve a maior } \\
\text { frequência de Burnout }(27,1 \%) \text {, enquanto o } \\
4^{\circ} \text { ano teve a menor }(2,1 \%) \text {. Os alunos }\end{array}$ & A \\
\hline
\end{tabular}




\begin{tabular}{|c|c|c|c|c|c|c|}
\hline & & & & & $\begin{array}{l}\text { apresentaram notas altas em exaustão } \\
\text { emocional }(63,2 \%) \text {. Houve correlação } \\
\text { significativa entre exaustão emocional e } \\
\text { descrença }\end{array}$ & \\
\hline $\begin{array}{l}\text { Grace, M. } \\
\text { K.; } 2018\end{array}$ & EUA & Longitudinal & $\begin{array}{l}286 \\
\text { pessoas }\end{array}$ & $\begin{array}{l}\text { Investigar a relação } \\
\text { entre problemas de } \\
\text { saúde mental e } \\
\text { interesse pela carreira } \\
\text { médica entre estudantes } \\
\text { de medicina e explorar } \\
\text { se essa associação varia } \\
\text { por gênero }\end{array}$ & $\begin{array}{l}\text { Os entrevistados que relataram mais } \\
\text { sintomas depressivos e níveis mais elevados } \\
\text { de Burnout apresentaram reduções } \\
\text { significativas no interesse da carreira } \\
\text { médica durante o período de estudo. Os } \\
\text { sintomas depressivos foram associados a } \\
\text { declínios mais acentuados no interesse pela } \\
\text { carreira médica entre as mulheres em } \\
\text { comparação aos homens, contudo a relação } \\
\text { entre Burnout e o interesse pela carreira } \\
\text { médica não variaram de modo significativo } \\
\text { por gênero }\end{array}$ & A \\
\hline $\begin{array}{l}\text { Fontana, } \\
\text { M. C. P. et } \\
\text { al.; } 2020\end{array}$ & Brasil & Transversal & $\begin{array}{l}121 \\
\text { internos }\end{array}$ & $\begin{array}{lr}\text { Identificar } & \text { a } \\
\text { prevalência da } & \text { SB } \\
\text { durante o internato e } & \\
\text { sua associação com } & \text { social } \\
\text { suporte } & \text { e } \\
\text { autorrelatado } & \\
\text { atividades } & \\
\text { extracurriculares em } \\
\text { uma faculdade de } \\
\text { medicina }\end{array}$ & $\begin{array}{l}\text { A prevalência total de SB foi } 57,5 \% \text { entre os } \\
\text { estudantes. Alta exaustão emocional foi } \\
\text { observada em } 33,1 \% \text { dos alunos e alta } \\
\text { despersonalização em } 45,7 \% \text {, enquanto } \\
36,2 \% \text { dos participantes demonstraram } \\
\text { reduzida realização pessoal. Indivíduos que } \\
\text { participaram de serviços comunitários } \\
\text { apresentaram menor frequência de alta } \\
\text { despersonalização. A SB não teve } \\
\text { associação com outros grupos de atividades } \\
\text { extracurriculares ou com grupos de suporte } \\
\text { social }\end{array}$ & A \\
\hline $\begin{array}{l}\text { Pagnin, D.; } \\
\text { Queiroz, } \\
\text { V.; } 2015\end{array}$ & Brasil & Transversal & $\begin{array}{l}193 \\
\text { estudantes }\end{array}$ & $\begin{array}{l}\text { Quantificar a influência } \\
\text { da SB e dificuldades de } \\
\text { sono na qualidade de } \\
\text { vida de estudantes de } \\
\text { medicina na fase pré- } \\
\text { clínica }\end{array}$ & $\begin{array}{l}\text { Houve uma diminuição da saúde física e } \\
\text { bem-estar psicológico com o aumento da } \\
\text { exaustão emocional, cinismo e da } \\
\text { dificuldade para dormir. Enquanto, a saúde } \\
\text { física, o bem-estar psicológico e as relações } \\
\text { sociais aumentaram quando houve uma } \\
\text { maior eficácia acadêmica. Assim, o bem- } \\
\text { estar físico e psicológico são associados de } \\
\text { modo negativo a exaustão emocional, } \\
\text { cinismo e dificuldades para dormir em } \\
\text { alunos na fase inicial da faculdade de } \\
\text { medicina. O esforço deve se direcionar para } \\
\text { o esgotamento e as dificuldades do sono. } \\
\text { Desse modo, se melhora a qualidade de vida } \\
\text { desses indivíduos }\end{array}$ & A \\
\hline $\begin{array}{l}\text { Calcides, } \\
\text { D. A. P. et } \\
\text { al.; } 2019\end{array}$ & Brasil & Transversal & $\begin{array}{l}184 \\
\text { internos }\end{array}$ & 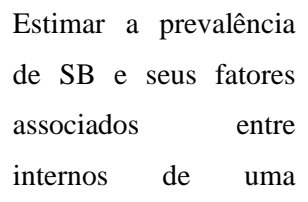 & $\begin{array}{l}\text { A prevalência de SB foi de } 35,9 \% \text { com base } \\
\text { no critério bidimensional (exaustão e } \\
\text { cinismo), foi maior naqueles que pensaram } \\
\text { em desistir do programa, dos que ficaram }\end{array}$ & A \\
\hline
\end{tabular}




\begin{tabular}{|c|c|c|c|c|c|c|}
\hline & & & & $\begin{array}{l}\text { Universidade pública } \\
\text { do nordeste do Brasil, } \\
\text { além de apurar a } \\
\text { contribuição do método } \\
\text { ou programa Balint na } \\
\text { sua prevenção }\end{array}$ & $\begin{array}{l}\text { insatisfeitos com as estratégias de ensino e } \\
\text { com seu desempenho e faziam uso de } \\
\text { drogas lícitas. A prevalência da SB } \\
\text { diminuiu e os fatores de vulnerabilidade } \\
\text { foram atenuados quando houve maior } \\
\text { frequência de alunos participando do } \\
\text { método Balint }\end{array}$ & \\
\hline $\begin{array}{l}\text { Cazolari, } \\
\text { P. G. et al.; } \\
2020\end{array}$ & Brasil & Transversal & $\begin{array}{l}302 \\
\text { estudantes }\end{array}$ & $\begin{array}{l}\text { Avaliar e descrever os } \\
\text { níveis de SB e } \\
\text { qualidade de vida dos } \\
\text { estudantes de medicina } \\
\text { da Universidade } \\
\text { Federal de São Paulo } \\
\text { (Unifesp) }\end{array}$ & $\begin{array}{l}\text { Os estudantes apresentaram baixo valor no } \\
\text { fator exaustão emocional e altos valores na } \\
\text { descrença e eficácia profissional, indicando } \\
\text { um Burnout entre baixo e moderado. } \\
\text { Observou-se também que estudantes do } \\
\text { gênero feminino apresentaram maior } \\
\text { tendência à exaustão emocional em } \\
\text { comparação ao gênero masculino. Foi } \\
\text { verificado que os possíveis fatores que } \\
\text { podem interferir na qualidade de vida dos } \\
\text { estudantes são: a carga horária excessiva de } \\
\text { atividades, modelo de ensino baseado em } \\
\text { aulas expositivas extensas, ausência de } \\
\text { estímulo e reconhecimento pelos seus } \\
\text { esforços }\end{array}$ & A \\
\hline $\begin{array}{l}\text { Gold, J. A. } \\
\text { et al.; } 2019\end{array}$ & $\begin{array}{l}\text { EUA } \\
\text { (China e } \\
\text { país do } \\
\text { Oriente } \\
\text { Médio) }\end{array}$ & $\begin{array}{l}\text { Estudo } \\
\text { exploratório }\end{array}$ & $\begin{array}{l}473 \\
\text { estudantes }\end{array}$ & $\begin{array}{l}\text { Examinar as taxas de } \\
\text { depressão em três } \\
\text { coortes internacionais } \\
\text { de estudantes de } \\
\text { medicina determinando } \\
\text { as variáveis que podem } \\
\text { explicar essas } \\
\text { diferenças }\end{array}$ & $\begin{array}{l}\text { Estudantes de medicina do país do Oriente } \\
\text { Médio apresentaram a maior taxa para } \\
\text { depressão }(41,1 \%) \text {, seguido da China } \\
(14,1 \%) \text { e dos Estados Unidos }(3,8 \%) \text {. } \\
\text { Observou-se que mais alunos na escola de } \\
\text { medicina do Oriente Médio tinham } \\
\text { necessidades de saúde mental não atendidas } \\
(50,8 \%) \text {. As variáveis demográficas não } \\
\text { predizem significativamente a depressão do } \\
\text { estudante de medicina; no entanto, a falta de } \\
\text { exercícios, as necessidades de saúde mental } \\
\text { não atendidas, o estresse e a exaustão } \\
\text { emocional previram quase metade da } \\
\text { depressão nessas coortes }\end{array}$ & A \\
\hline
\end{tabular}

Fonte: Autores, (2020).

No Quadro 2 se torna significativo observar as especificidades dos artigos selecionados para a amostra final, bem como o nível de evidência de cada um de acordo com o CASP adaptado. Nesta perspectiva, abaixo expomos com mais detalhes as discussões.

Não existe uma causa principal definida para o distúrbio, mas a soma de múltiplos elementos facilitam para o desencadeamento da $\mathrm{SB}$, tanto fatores extrínsecos, quanto intrínsecos. Os fatores extrínsecos são associados ao enaltecimento do curso de medicina e o quão exigente começa a ser a vida depois de iniciada na graduação do referido curso, no qual o estudante busca mais e mais a qualificação e destaque no mesmo, além da pressão de terceiros como pacientes, colegas e a sociedade (Mori, Valente e Nascimento, 2012). 
Os fatores intrínsecos impostos pelo próprio estudante, em que vai estar exposto a estressores ao longo de sua formação, devido a razões como excessiva carga de estudos, pouco tempo para lazer e para a família, estressores que podem ser agravados pelos tipos de personalidade com traços obsessivos, obstinação, perfeccionismo que, se persistentes, podem desencadear a síndrome (Barbosa et al., 2018).

De acordo com Santen et al. (2010), na faculdade de medicina de Vanderbilt - Estados Unidos da América (EUA), houve a presença de grau moderado ou alto de Burnout em $21 \%$ da classe do primeiro ano, $41 \%$ no segundo ano, o terceiro ano com o maior índice de $43 \%$ da classe e o quarto ano pesquisado com $31 \%$ da classe apresentando tal síndrome. Outro estudo apontou que alunos do terceiro ano demonstraram o maior índice $(27,1 \%)$ de SB em comparação com os demais alunos participantes, eles ainda indicaram a maior proporção de exaustão emocional positiva (15,9\%) (Barbosa et al., 2018).

É um problema que se mantém ativo com o passar do tempo, pois Fitzpatrick et al. (2019) afirma que o esgotamento é comum em estudantes de medicina. Já Pacheco et al. (2017) salienta que grande parte dos estudantes de medicina brasileiros possuem múltiplos problemas de saúde mental (PSM). Abrangendo assim, estresse psicológico, ansiedade, depressão, esgotamento, distúrbios do padrão de sono, distúrbios alimentares e uso excessivo de bebidas alcoólicas.

A pesquisa de Pagnin e Queiroz (2015) indicou que os estudantes de medicina se demonstraram exaustos emocionalmente e com dificuldades para dormir. Diante disso, foi salientado que a qualidade de vida dos futuros médicos é afetada pela exaustão e dificuldade citadas anteriormente, ou seja, apesar de aprenderem a promover um atendimento buscando a melhora da saúde de seus pacientes, por vezes, descuidam-se de um cuidado para com a saúde deles mesmos.

Ávila (2014) observou que em um grupo de alunos pesquisados a maior parte é do sexo feminino, considerada uma tendência brasileira dos últimos anos nas escolas de medicina, bem como, de novos inscritos nos Conselhos Médicos. Dessa forma, observa-se que é no sexo feminino que prevalece a SB em comparação aos homens (Barbosa et al., 2018). Nesta perspectiva, Grace (2018) destaca também que as mulheres apresentaram uma sintomatologia depressiva maior e índices mais altos de SB em comparação ao sexo oposto. Destarte, os sintomas depressivos e SB variam de forma significativa por gênero.

Pastura et al. (2018) relata o grupo Balint (GB) como uma ação em conjunto com a liderança de um psicanalista para discussão dos pacientes, cujo foco é na relação médico-paciente e nas reações do médico. Calcides et al. (2019) faz referência a uma menor prevalência de SB, como também uma frequência mais baixa de uso de drogas lícitas naqueles que demonstraram uma maior satisfação com o desempenho acadêmico e as formas de ensino, tendo uma participação superior no GB e a convicção de que esse processo auxilia na sua formação como médico.

Cazolari et al. (2020) expõe em seu estudo que os prováveis fatores de interferência nos estudantes de medicina estão centrados na carga horária excessiva de atividades, modelo de ensino baseado em aulas expositivas extensas, ausência de estímulo e reconhecimento pelos seus esforços.

Gold et al. (2019) concluiu que estudantes de medicina do país do Oriente Médio apresentaram a maior taxa de depressão, seguida pela China e EUA, porém em exaustão emocional, um dos três sintomas principais do Burnout, os EUA alcançaram o índice mais alto. Ele também considera que tanto a depressão como a exaustão emocional estão ligadas a falta de exercício, necessidades e saúde mental não atendidas e estresse. Maia et al. (2012) e Cecil et al. (2014) corroboram na declaração de que para provocar um menor nível de SB nos futuros médicos, se faz necessário um estilo de vida mais saudável com práticas de exercícios físicos e ingerir alimentos mais naturais.

Durante o curso de medicina, as mudanças provocadas pelo estilo de vida de estresses e cobranças têm diminuído a qualidade de vida desses graduandos (Fiedler, 2008). Corroborando com esse fato, Mustafa (2015) afirma que os comportamentos na área da saúde são considerados preditivos de esgotamento. Por isso, certas atitudes como atividade física são consideradas protetoras e, portanto, devem ser estabelecidas no início da vida universitária para prevenir o desenvolvimento dessa síndrome. 
Segundo Fontana et al. (2020), 57,5\% dos residentes de medicina da Faculdade de Ciências Médicas da Santa Casa de São Paulo apresentaram a SB. Para a elaboração da pesquisa foi utilizado o Maslach Burnout Inventory - Serviços Humanos para avaliação de SB, um instrumento validado com três subescalas: exaustão emocional, despersonalização e realização pessoal. Nesse contexto, entre os acadêmicos de medicina acometidos há características como alta exaustão emocional, despersonalização e realização pessoal reduzida (três dimensões da síndrome). Isso acontece como reflexo da exposição crônica a situações de estresse e pressão pela sobrecarga de obrigações no ambiente de estágio (internato), no qual atinge de forma brusca as relações interpessoais dos estudantes, interferindo negativamente na qualidade dos atendimentos para os enfermos, além do relacionamento com a equipe de saúde. Esse quadro causa um ciclo vicioso de frustrações pessoais, desgaste físico e mental.

O estudo relatou que dependendo do estado psicossocial e da atividade extracurricular exercida pelo acadêmico, essa síndrome pode ser reduzida ou exacerbada. Ademais, houve menor prevalência de alta despersonalização entre os acadêmicos residentes que exerciam atividades extracurriculares ligadas a comunidade, fruto do altruísmo praticado que auxilia na humanização do atendimento. O apoio psicossocial também é crucial para uma reversão da SB.

A SB pode ter início na época da faculdade de medicina, prossegue durante toda a residência e, por fim, cresce na rotina de médicos em atividade (Romani e Ashkar, 2014). Shanafelt et al. (2012) apontou que dos 7.288 médicos que participaram do estudo, 45,8\% deles relataram pelo menos um sintoma de SB e o estudo de Balch et al. (2011) determinou que processos de negligência médica foram relacionados diretamente a SB, depressão e pensamentos de suicídio entre cirurgiões. Assim, como apontou Pacheco et al. (2017) os PSM são de alta prevalência entre os estudantes no Brasil e intervenções baseadas em evidências, bem como, os apoios psicossociais são cruciais para a promoção da saúde mental dos alunos e futuros médicos do país.

\section{Conclusão}

O presente estudo demonstrou que o acadêmico de medicina enfrenta uma grade curricular extensa, por consequência com grande carga de informações e meio intenso de produtividade, que pode gerar um esgotamento físico, mental e afetar o desempenho durante a sua formação. Vale salientar que há uma cobrança ao estudante ser e portar-se como um bom profissional. Sendo esses alguns agravantes que aumentam a possibilidade do desenvolvimento da SB nos alunos.

Observa-se que os acadêmicos do $3^{\circ}$ ano são os mais afetados, tendo as mulheres uma maior prevalência ao apresentar sintomas de depressão, como também de desenvolver a SB. Vale ressaltar ainda, que elas estão em maior quantidade nos cursos médicos brasileiros, bem como nas novas inscrições dos Conselhos de Medicina.

Percebe-se que para amenizar tal síndrome, faz-se necessário estar atento a uma melhora na qualidade de vida adquirida com práticas de exercícios físicos diariamente, uma alimentação mais saudável, tempo maior para lazer, participar do GB, ter apoio psicossocial, exercer atividades extracurriculares ligadas à comunidade, dentre outras ações protetoras. É possível acrescentar que tais ações trarão benefícios para o acadêmico na sua atuação, cuidando primeiramente de si para promover a saúde do outro.

Desse modo, foi relevante estudar a SB pelos conhecimentos adquiridos através das informações sistematizadas e se sugere o desenvolvimento de outros estudos relacionados a essa temática que venham colaborar efetivamente para o benefício tanto dos estudantes de medicina, quanto da sociedade em geral, pois estudantes de medicina e médicos saudáveis proporcionarão um atendimento ao público de mais qualidade, auxiliando na prevenção e tratamento de doenças.

Portanto, recomenda-se como sugestão para trabalhos futuros averiguar a correlação entre a SB desenvolvida entre os acadêmicos de medicina e médicos e outras profissões, visto que demonstrará se são níveis diferentes de Burnout dependendo da profissão e o porquê dessa diferença, se houver. Outro tema pertinente para investigação é de que modo a pandemia da 
COVID-19 afetou a saúde psicológica dos alunos de medicina e dos médicos, aprofundando-se em entender se a pandemia aumentou o nível de SB naqueles que já tinham, se a pandemia foi um fator predisponente para o aparecimento da mesma ou se de algum modo desencadeou-se outras doenças mentais associadas.

\section{Referências}

Ávila, R. C. (2014). Formação das Mulheres nas Escolas de Medicina. Revista Brasileira de Educação Médica, $38(1), 142-149$.

Balch, C. M. et al. (2011). Personal Consequences of Malpractice Lawsuits in American Surgeons. Journal of the American College of Surgeons, 213(5), 657667.

Barbosa, M. L. et al. (2018). Burnout Prevalence and Associated Factors among Brazilian Medical Students. Clinical Practice and Epidemiology in Mental Health, 14, 188-195.

Benevides-Pereira, A. M. T. (2012). Considerações sobre a Síndrome de Burnout e seu Impacto no Ensino. Boletim de Psicologia, 62(137), 155-168.

Calcides, D. A. P. et al. (2019). Burnout Syndrome in Medical Internship Students and its Prevention with Balint Group. Revista da Associação Médica Brasileira, 65(11), 1362-1367.

Cazolari, P. G. et al. (2020). Níveis de Burnout e Bem-Estar de Estudantes de Medicina: Um Estudo Transversal. Revista Brasileira de Educação Médica, 44(4), 1-8.

Cecil, J. et al. (2014). Behaviour and Burnout in Medical Students. Medical Education Online, 19, 1-9.

Ercole, F. F., Melo, L. S., \& Alcoforado, C. L. G. C. (2014). Integrative Review Versus Systematic Review. [Editorial]. Revista Mineira de Enfermagem, $18(1), 12-14$.

Feodrippe, A. L. O., Brandão M. C. F., \& Valente T. C. O. (2013). Qualidade de Vida de Estudantes de Medicina: Uma Revisão. Revista Brasileira de Educação Médica, 37(3).

Fiedler, P. T. (2008). Avaliação da Qualidade de Vida do Estudante de Medicina e da Influência Exercida pela Formação Acadêmica. Tese (Doutorado em Ciências) - Faculdade de Medicina. Universidade de São Paulo.

Fitzpatrick, O. et al. (2018). Prevalence and Relationship between Burnout and Depression in Our Future Doctors: A Cross-Sectional Study in a Cohort of Preclinical and Clinical Medical Students in Ireland. BMJ Open, 9, 1-7.

Fontana, M. C. P. (2020). Burnout Syndrome, Extracurricular Activities and Social Support among Brazilian Internship Medical Students: A Cross-Sectional Analysis. BMC Medical Education, 20(81), 3-9.

Gold, J. A. et al. (2019). Medical Student Depression and Its Correlates Across Three International Medical Schools. World Journal of Psychiatry, 9(4), 65-77.

Gonçalves, M. B., Benevides-Pereira, A. M. T. (2009). Considerações sobre o Ensino Médico no Brasil: Consequências Afetivo-Emocionais nos Estudantes. Revista Brasileira de Educação Médica, 33(3), 493-504.

Grace, M. K. (2018). Depressive Symptoms, Burnout, and Declining Medical Career Interest Among Undergraduate Premedical Students. International Journal of Medical Education, 9, 302-308.

Maia, D. A. C., et al. (2012). Cadernos ESP/CE, 6(2), 50-59.

Moreira, H. A., Souza, K. N., \& Yamaguchi, M. U. (2018). Síndrome de Burnout em Médicos: Uma Revisão Sistemática. Revista Brasileira de Saúde Ocupacional, 43, 1-11.

Mori, M. O., Valente, T. C. O., \& Nascimento, L. F. C. (2012). Síndrome de Burnout e Rendimento Acadêmico em Estudantes da Primeira à Quarta Série de um Curso de Graduação em Medicina. Revista Brasileira de Educação Médica, 36(4), 536-540.

Mustafa, O. M. (2015). Health Behaviors and Personality in Burnout: A Third Dimension. Medical Education Online, 20(1), 1-6.

Pacheco, J. P. et al. (2017). Mental Health Problems Among Medical Students in Brazil: A Systematic Review and Meta-Analysis. Revista Brasileira de Psiquiatria, 39, 369-378.

Pagnin, D., Queiroz, V. (2015). Influence of Burnout and Sleep Difficulties on the Quality of Life Among Medical Students. Springer Plus, 4(676), 1-7.

Pastura, P. S. V. C. et al. (2019). Do Burnout à Estratégia de Grupo na Perspectiva Balint: Experiência com Residentes de Pediatria de um Hospital Terciário. Revista Brasileira de Educação Médica, 43(2), 32-39.

Romani, M., Ashkar, K. (2014). Burnout Among Physicians. Libyan Journal of Medicine, 9, 1-6.

Santen, S. A. et al. (2010). Burnout in Medical Students: Examining the Prevalence and Associated Factors. Southern Medical Association, 103(8), 758-763.

Shanafelt, T. D. et al. (2012). Burnout and Satisfaction with Work-Life Balance among US Physicians Relative to the General US Population. JAMA Internal Medicine, 172(18), 1377-1385. 
Research, Society and Development, v. 10, n. 5, e15210514697, 2021

(CC BY 4.0) | ISSN 2525-3409 | DOI: http://dx.doi.org/10.33448/rsd-v10i5.14697

Silva, F. B. et al. (2009). Atitudes Frente a Fontes de Tensão do Curso Médico: Um Estudo Exploratório com Alunos do Segundo e do Sexto Ano. Revista Brasileira de Educação Médica, 33(2), 230-239. 